\title{
The Circadian Effects on Postural Stability in Young Adults
}

\author{
Sung Min Son \\ Department of Physical Therapy, College of Health Science, Cheongju University, Cheongju, Korea
}

Purpose: Few studies have addressed the effect of diurnal circadian rhythms on postural stability, and thus the aim of the present study was to examine circadian effects on static and dynamic postural stability in young adults.

Methods: Twenty-four subjects (9 men, 11 women: age $=22.20 \pm 1.77$, height $=167.20 \pm 10.47$, weight $=59.85 \pm 10.66$ ) from a university community volunteered for this study. Static and dynamic balance testing, which recorded using a Good Balance system (Good Balance, Metitur Ltd, Finland) was conducted at 9:00, 13:00, and 17:00 hours on two consecutive days, and the sequencing of static and dynamic balance tests were randomized. Results were analyzed using the non-parametric one-way repeated Friedman test in SPSS version 18.0 (SPSS Inc., Chicago, IL, USA), and variable found to be significant were subjected to Wilcoxon post hoc testing.

Results: Static and dynamic balance showed significant difference at the three times assessments (test at 9:00, 13:00, and 17:00) during circadian. In the post hoc test of static (anteroposterior distance, mediolateral distance and COP (center of pressure) velocity) and dynamic balance (performance time), 13:00 was the longer and faster than 9:00.

Conclusion: These results indicated that control of postural balance is influenced by diurnal circadian rhythms, and confirm that control of postural balance is more effective and better performance in the 09:00 hours than 13:00 hours or 17:00 hours.

Keywords: Time-of-day, Postural control, Static balance, Dynamic balance

\section{Introduction}

Postural stability is defined as the ability to control position in order to maintain one's center of gravity or equilibrium within the limits of stability, over a base of support during functional activities and to compensate for internal and external perturbations. ${ }^{1,2}$ It is influenced by a range of various sensory and motor functions, including the visual and vestibular systems, proprioceptive information, and muscular strength., ${ }^{3,4}$ Various studies have reported changes in postural stability related to the neuromuscular system (fatigue due to general and local exercise) ${ }^{2,5}$ and the cognitive system (e.g., mental fatigue due to a demanding sustained-attention task), ${ }^{6}$ which can impact motor activity.

It is well known and certain that biological rhythms may be influenced by time-of-day, possibly due to variations caused by hormonal changes, body temperature, perceptions of fatigue, somatosensory input, muscular strength, and other factors. ${ }^{7.8}$ Diurnal cir-

Received May 22, 2017 Revised Jun 22, 2017

Accepted Jun 28, 2017

Corresponding author Sung Min Son

E-mail ssm0417@hanmail.net cadian rhythms are influenced many physiological functions in the human organism, such as, cognitive function and metabolic state, and cause fluctuations in physiological functions. ${ }^{910}$ In other words, diurnal circadian rhythms may affect functional ability to perform various types of motor tasks, including maintenance of postural stability. However, few studies have addressed the effect of diurnal circadian rhythms on postural stability, and thus, this study was undertaken to investigate circadian effects on static and dynamic postural stability in young adults.

\section{Methods}

\section{Subjects}

Twenty subjects ( 9 men, 11 women: age $=22.20 \pm 1.77$, height $=$ $167.20 \pm 10.47$, weight $=59.85 \pm 10.66$ ) from a university community volunteered for this study. All subjects provided their informed consent before participating in the study. Subjects were free from

Copylight (C)2017 The Korea Society of Physical Therapy

This is an Open Access article distribute under the terms of the Creative Commons Attribution Non-commercial License (Http:// creativecommons.org/license/by-nc/4.o.) which permits unrestricted non-commercial use, distribution, and reproduction in any medium, provided the original work is properly cited. 
Table 1. Comparison of static and dynamic balance variables at the three testing time

\begin{tabular}{|c|c|c|c|c|c|c|}
\hline & & & & & f Day & \\
\hline & & & 9:00 AM & 13:00 PM & $17: 00 \mathrm{PM}$ & $\chi^{2}$ Chi-square ( $p$ value) \\
\hline Static Balance & AP Distance & Row & $130.33 \pm 69.07$ & $160.17 \pm 47.97$ & $153.43 \pm 70.51$ & $6.7(0.035)$ \\
\hline & & Mean Rank & $1.55^{*}$ & 2.35 & 2.1 & \\
\hline & ML Distance & Row & $8.44 \pm 7.58$ & $15.05 \pm 11.10$ & $11.15 \pm 6.07$ & $8.38(0.015)$ \\
\hline & & Mean Rank & $1.48^{*, \neq}$ & 2.28 & 2.25 & \\
\hline & COP Velocity & Row & $6.82 \pm 2.41$ & $11.35 \pm 9.40$ & $9.15 \pm 8.56$ & $6.56(0.038)$ \\
\hline & & Mean Rank & $1.68^{*}$ & 2.45 & 1.88 & \\
\hline Dynamic Balance & Performance Time & Row & $17.55 \pm 4.47$ & $24.77 \pm 9.49$ & $27.77 \pm 20.43$ & $8.68(0.013)$ \\
\hline & & Mean Rank & $1.48^{*, *}$ & 2.35 & 2.17 & \\
\hline & Total Distance $(\mathrm{cm})$ & Row & $21.81 \pm 9.93$ & $30.20 \pm 16.08$ & $27.62 \pm 13.13$ & $4.9(0.086)$ \\
\hline & & Mean Rank & 1.6 & 2.25 & 2.15 & \\
\hline
\end{tabular}

*significant difference compared with 13:00 PM $(p<0.05)$.

"significant difference compared with 17:00 PM $(p<0.05)$.

lower limb injury (e.g. strain, sprain, surgery, or fracture) and any neurologic or vestibular impairment that might influence postural stability for a period of 6 months prior to study commencement. Additionally, subjects were asked to sleep as normal and not to consume alcohol for 24 hours before testing day.

\section{Experimental methods}

Static and dynamic balance tests were conducted using a Good Balance system (Good Balance, Metitur Ltd, Finland), which consists of a portable equilateral triangular force platform $(800 \times 800 \times 800$ $\mathrm{mm}$ ) equipped with strain gauge transducers connected to a threechannel DC amplifier and a 12-byte analogue-to digital (A/D) converter connected to a computer. Indexes of the Good Balance system representative of static and dynamic balance ability, including shifts in the center of pressure (COP) in the mediolateral and anteroposterior directions, and the path of COP, were used to measure postural stability.

To measure static balance (anteroposterior and mediolateral direction, and COP velocity) under stable surface condition, subjects stood on the force plate with legs spread at shoulder width and then looked at a number on a monitor for 30 seconds, and to measure dynamic balance (performance time and total distance), nine boxes, which consist of eight peripheral targets and one central target, were shown on the monitor. Subjects were asked to shift their COP toward a target when one of the peripheral targets was presented at random, and then to shift back to the central target. Laboratory testing was performed at 9:00, 13:00, and 17:00 hours on two con- secutive days, and static and dynamic balance tests were randomized. Subjects were allowed to familiarize themselves with the balance test beforehand to minimize learning effects. Three repeat measurements were taken at each of the six test sessions, and average values were entered into the analysis. A rest period of three minutes was allowed between measurements at individual sessions.

\section{Statistical analysis}

Results were analyzed using the non-parametric one-way repeated Friedman test in SPSS version 18.0 (SPSS Inc., Chicago, IL, USA), and variable found to be significant were subjected to Wilcoxon post hoc testing. Statistical significance was accepted for values $<0.05$.

\section{Result}

Static balance, which was measured anteroposterior and mediolateral distances, and COP velocity, were found significantly different at the 3 assessments during circadian, and post hoc testing showed anteroposterior and mediolateral distance, and COP velocity, was longer and faster at 13:00 hours than 9:00 hours.

Dynamic balance, that is performance time, was also revealed significant difference among three assessments, and post hoc testing showed performance time at 13:00 hours was the longer than 9:00 hours. However, total distance was not significant difference among 3 assessments. 


\section{Discussion}

In this study, we investigated the effect of time of day on static and dynamic postural stability in young adults. Our results indicated that control of static balance (AP distance, ML distance and COP velocity) and dynamic balance (performance time and total distance) were more better at 9:00 hours than 13:00 hours and 17:00 hours. Accordingly, our results indicate diurnal circadian rhythms influence control of postural balance.

More specifically, static and dynamic balance measures at 9:00 hours revealed smaller distances, velocities and shorter performance times for static and dynamic parameters than at 13:00 hours, indicating that control of postural balance was better in the morning than in the early afternoon. The mechanism responsible for these changes in postural balance has not been determined. However, it is possible that motor performance and postural balance might be influenced by circadian rhythms, which involve cognitive functions, sensory inputs and muscle activation. Considering postural balance with physical activity or attentional loading, results of our study could be related to the increase of postural instability with cognitive and generalized metabolic fatigue-induced manipulation during time of day. It has been previously suggested elevated levels of physical activity or demanding cognitive functions alter motor performance and postural balance. ${ }^{11-14}$ Baccourch et al. ${ }^{15}$ also reported that attentional capacities were better at 10:00 hours and 18:00 hours in comparison with 14:00 hours during time-of-day. This result is consistent with our results, which mean rank of postural control was lower at 13:00 hours in comparison with 9:00 hours and 17:00 hours.

These results indicated that control of postural balance is influenced by diurnal circadian rhythms, and confirm that control of postural balance is more effective and better performance in the 09:00 hours than 13:00 hours or 17:00 hours. Therefore, when choosing to use postural balance test for comparisons across days or group, it is meant that test might need to maintain consistency of the testing time. When interpreting the data, several limitation of the present study warrant consideration. First, the amount of physical activities were not controlled for, although subjects were asked to refrain from undue exercise prior to testing session. Second, the study was conducted on healthy and young age subject, and thus, additional studies are required on other age groups. Conduct of fu- ture studies to include the factors mentioned above will be required.

\section{Acknowledgements}

This work was supported by the research grant of Cheongju University (2016.09.01 -2018.08.31).

\section{Reference}

1. Pollock AS, Rowe PJ, Paul JP et al. What is balance? Clin Rehabil. 2000; 14(4):402-6.

2. Ruhe A, Fejer R, Walker B. The test-retest reliability of centre of pressure measures in bipedal static task conditions--a systematic review of the literature. Gait Posture. 2010;32(4):436-45.

3. Loram ID, Lakie M. Direct measurement of human ankle stiffness during quiet standing: The intrinsic mechanical stiffness is insufficient for stability. J Physiol. 2002;545(Pt 3):1041-53.

4. Lord SR, Clark RD, Webster IW. Postural stability and associated physiological factors in a population of aged persons. J Gerontol. 1991; 46(3):M69-76.

5. Sparto PJ, Parnianpour M, Reinsel TE et al. The effect of fatigue on multijoint kinematics, coordination, and postural stability during a repetitive lifting test. J Orthop Sports Phys Ther. 1997;25(1):3-12.

6. Deschamps T, Magnard J, Cornu C. Postural control as a function of time-of-day: influence of a prior strenuous running exercise or demanding sustained-attention task. J Neuroeng Rehabil. 2013;10:26.

7. Guariglia DA, Pereira LM, Dias JM et al. Time-of-day effect on hip flexibility associated with the modified sit-and- reach test in males. Int J Sports Med. 2011;32(12):947-52.

8. Souissi N, Bessot N, Chamari K et al. Effect of time of day on aerobic contribution to the 30 -s wingate test performance. Chronobiol Int. 2007;24(4):739-48.

9. Kwon YH. Influence of time-of-day on respiratory function in normal healthy subjects. J Korean Soc Phys Ther. 2013;25(6):374-8.

10. Winget CM, DeRoshia CW, Holley DC. Circadian rhythms and athletic performance. Med Sci Sports Exerc. 1985;17(5):498-516.

11. Helbostad JL, Sturnieks DL, Menant J et al. Consequences of lower extremity and trunk muscle fatigue on balance and functional tasks in older people: a systematic literature review. BMC Geriatr. 2010;10:56.

12. Papa EV, Foreman KB, Dibble LE. Effects of age and acute muscle fatigue on reactive postural control in healthy adults. Clin Biomech (Bristol, Avon). 2015;30(10):1108-13.

13. Stins JF, Kempe CL, Hagenaars MA et al. Attention and postural control in patients with conversion paresis. J Psychosom Res. 2015;78(3):249-54.

14. Hoyer D, Clairambault J. Rhythms from seconds to days. Physiological importance and therapeutic implications. IEEE Eng Med Biol Mag. 2007;26(6):12-3.

15. Baccouch R, Zarrouk N, Chtourou H et al. Time-of-day effects on postural control and attentional capacities in children. Physiol Behav. 2015;142:146-51. 